\title{
Quantum cascade celebration
}

\author{
Twenty years ago, researchers at Bell Labs in the USA stunned the optics world by reporting a new type of \\ semiconductor laser - the quantum cascade laser. This laser transformed mid- and far-infrared photonics.
}

This year marks the 20th anniversary of the first experimental demonstration of the quantum cascade laser (QCL) by a team at Bell Labs led by Federico Capasso (now a professor at Harvard University). The breakthrough was announced in a paper reporting pulsed lasing action at $4.2 \mu \mathrm{m}$ that was published on 22 April 1994 in Science (Faist, J. et al. Science 264, 553-556; 1994). It brought instant fame to the paper's six authors: Jerome Faist, Federico Capasso, Deborah Sivco, Carlo Sirtori, Albert Hutchinson and Alfred Cho.

In a conventional semiconductor $\mathrm{p}-\mathrm{n}$ junction diode laser, light is generated when injected electrons in the conduction band recombine with holes in the valence band. In contrast, in a triumph of quantum and semiconductor engineering, QCLs

exploit optical transitions between subband states formed within semiconductor multiquantum wells.

An appealing feature of this approach is that the emission wavelength can be controlled by tailoring the design of the multi-quantum wells. As a result, QCLs have become invaluable devices that span the wavelength range from mid-infrared to terahertz $(\mathrm{THz})$ waves. In contrast, diode lasers cannot produce radiation in this wavelength range.

The concept of intersubband light amplification - a key process for lasing in QCLs - had actually been proposed 23 years earlier by R. F. Kazarinov and R. A. Suris (Sov. Phys. Semicond. 5, 707-709; 1971). However, it took more than two decades to overcome a variety of technological and scientific challenges. A particularly thorny problem was how to produce the high population inversion required to generate laser action in a system for which the lifetime of electrons in an excited energy state is lower than those of diode lasers and other solid-state systems by a few and many orders of magnitude, respectively.

The researchers at Bell Labs initially thought that using an optical pumping scheme would be the easiest way to realize laser action between the electronic states of a quantum well. However, they met with almost complete failure over the two years they followed this strategy.

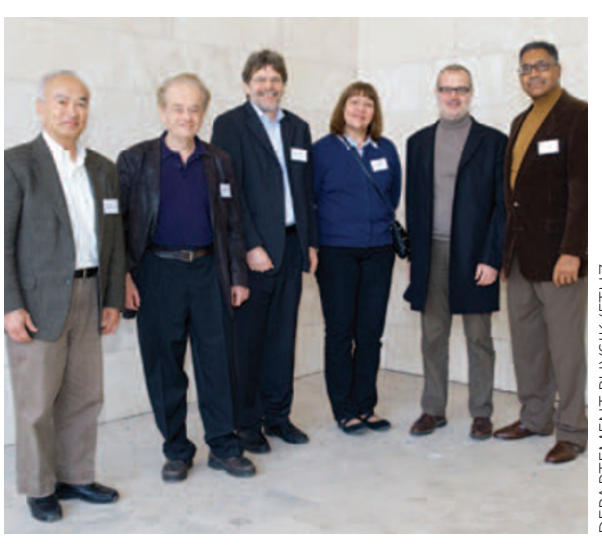

they are attracting the interest of major industrial players." The wavelength range now spans an incredible two decades of frequency $(2.6-250 \mu \mathrm{m})$. Very large output powers have been achieved, as well as narrow linewidths with a very high spectral purity. Furthermore, QCLs can support very wide tuning ranges as well as frequency comb operation.

As convenient sources of mid- and far-infrared light, QCLs are extremely useful for spectroscopic applications, such as chemical sensing. Robert Curl, Nobel Laureate in Chemistry in 1996 and one of the most active users of QCLs, states, "In the short space of 15 years

The original team members who exploited QCLs at Bell Labs. From the left: Alfred Y. Cho, Federico Capasso, Jérôme Faist, Debbie Sivco, Carlo Sirtori and Al Hutchinson.

The turning point came when they adopted an electron injection scheme that used a tunnelling current to resonantly inject electrons from a reservoir into the upper state of the laser transition. They designed an active region for electroluminescence and, in August 1993, obtained clear spectra with a narrow peak centred at a wavelength near $5 \mu \mathrm{m}$, which was evidence of population inversion. Capasso nostalgically recalls the first occurrence of lasing in a QCL, "On January 14, 1994, a Friday night, I was getting ready to go to the Metropolitan Opera in New York when I got a phone call from Jérôme Faist 'Federico, it lases!'”

Over just a few years, the team achieved room-temperature, pulsed operation, produced single-mode QCLs based on distributed feedback, and extended the operation wavelength from $3.4 \mu \mathrm{m}$ to about $20 \mu \mathrm{m}$. The impact of QCLs was immediate and very strong in the mid- and farinfrared community.

"Just few years after the birth of QCLs, the technology of low-gap laser diodes was supplanted by this new type of unipolar device, particularly for wavelengths longer than $4 \mu \mathrm{m}$ ", Carlo Sirtori, now a professor at Université Paris-Diderot, commented.

Faist added, "QCLs have moved from a lab curiosity to the marketplace, where since their first demonstration, quantum cascade lasers have become the most useful sources of tunable mid-infrared laser radiation" (Curl, R. F. et al. Chem. Phys. Lett. 487, 1-18; 2010).

Although it still remains to be fully explored, another interesting application is QCL-based sensors for the 'Internet of things'. Capasso explained, "the Internet of things will integrate sensor networks, thus providing unprecedented functionalities in terms of real-time, adaptive monitoring and data processing in many areas, including sensing and imaging." He envisions that mid-infrared QCL systems, including mid-infrared photonic integrated circuits with on-chip active and passive components, will have a major impact in these areas.

Going forward, what is the next great challenge for QCLs? Is it possible to achieve $1 \mathrm{~mW}$ or more of pulsed optical power in a terahertz QCL at room temperature? Can the emission wavelength of continuous-wave, room-temperature QCLs be extended to the short-wavelength range $3-3.5 \mu \mathrm{m}$ ?

Perhaps some of these questions will be answered at the International Quantum Cascade Laser School and Workshop, which will be held in Italy over September 7-12 (http://www.iqclsw2014. cnr.it/). The leading experts of QCLs will celebrate the 20th anniversary of QCLs by addressing fundamental physics and novel phenomena as well as new outstanding scientific, technological and application developments of QCLs from the mid- to far-infrared spectral region. 